\title{
ESTRESORES LABORALES DEL PERSONAL DE ENFERMERÍA DE UN HOSPITAL DE ALTA ESPECIALIDAD EN MÉXICO
}

WORKPLACE STRESSORS OF NURSING STAFF AT A HOSPITAL IN HIGH SPECIALTY IN MEXICO

Recibido agosto 2016 Aceptado diciembre 2016

Correspondencia: María Fernanda Ramírez López ramirezmarifer92@gmail.com
Autores:

María Fernanda Ramírez López

Pasante de la Licenciatura en Enfermería

Universidad Panamericana

Ciudad de México.

Angélica Guadalupe Dávalos Alcázar Coordinadora de Investigación en Enfermería Instituto Nacional de Neurología y Neurocirugía Manuel Velasco Suárez.

Rebeca Victoria Ochoa Facultad de Ciencias de la Salud

Escuela de Enfermería, Universidad Panamericana Ciudad de México.

Ximena del Carmen Rubilar Araya Facultad de Ciencias de la Salud

Escuela de Enfermería, Universidad Panamericana Ciudad de México.

Palabras clave: nivel de estrés, estresores laborales, enfermería.

Keywords: level of stress, workplace stressors, nursing. 
RESUMEN

Introducción: el profesional de enfermería se expone diariamente con situaciones que pueden ser generadoras de estrés como lo es la confrontación con la muerte, el sufrimiento de las personas, la carga de trabajo y los posibles conflictos con el personal interdisciplinario. La presente investigación se realizó en el Instituto Nacional de Neurología y Neurocirugía Manuel Velasco Suárez ubicado al sur de la Ciudad de México el cual brinda atención de tercer nivel.

Objetivo: identificar el nivel de estrés y los estresores laborales a los que están expuestos los enfermeros.

Metodología: se realizó un estudio transversal y descriptivo, la muestra fueron 50 enfermeros adscritos a los servicios de hospitalización los cuales contestaron un instrumento autoaplicacble conformado por 32 preguntas cerradas en escala de Lickert.

Resultados: el 52\% de los enfermeros tiene un nivel de estrés moderado, el $46 \%$ tiene un nivel de estrés bajo frente al 2\% que tiene un nivel de estrés alto. Los tres agentes estresores con medias más altas fueron en primer lugar realizar procedimientos dolorosos para el paciente $(X=1.88)$, en segundo lugar mirar sufrir a un paciente y terminar adolorido después de una jornada de trabajo $(X=1.82)$, el tercer lugar lo ocupa la falta de personal adecuado para cubrir la unidad $(X=1.7)$.

Conclusiones: en este estudio se logró identificar los agentes estresores laborales más comunes así como el nivel de estrés al que está expuesto el personal de enfermería de las áreas de hospitalización, el cual es producto de las situaciones que viven a diario para realizar sus intervenciones a favor del paciente esto lo expone a tener un nivel de estrés moderado, lo cual podría perjudicar su salud, pudiendo provocar una disminución en el desempeño sobre la calidad de las tareas que se realizan.

Palabras clave: nivel de estrés, estresores laborales, enfermería.
ABSTRACT

Introduction: every day the nursing professional is exposed go situations that can trigger stress as when dealing with death, the suffering of people, the workload and the possible conflicts when dealing with interdisciplinary staff. This research was conducted at the Instituto Nacional de Neurología y Neurocirugía Manuel Velasco Suárez, which provides the third-level of medical care and is located in the south of Mexico City.

Objective: to identify the level of stress and the workplace stressors which nurses are exposed to.

Methodology: through a cross-sectional descriptive survey, the sample was of 50 nurses who answered a selfadministered questionnaire composed by 32 closed questions in Lickert scale. The Nurses are attached to the hospitalization services.

Results: 52\% of the nurses have a moderate level of stress; the $46 \%$ have a low level of stress against the $2 \%$ that have a high level of stress. The three highest average stressors were in first place painful procedures for the patient ( $X=1.88$ ) secondly, to withess the suffering of a patient and body aches after a day of work $(X=$ 1.82) in third place is the lack of proper staff to cover the health care unit $(X=1.7)$.

Conclusions: it was possible to identify the most common workplace stressors as well as the level of stress which the nursing personnel is exposed to in hospitalization units. Nurses cope with stress in different moments of their daily work activities. When working for the patients they are more likely to have a moderate level of stress, which might be harmful for their health, and may be a cause of poor performance on the tasks they perform.

Key words: level of stress, workplace stressors, nursing.

\section{6}

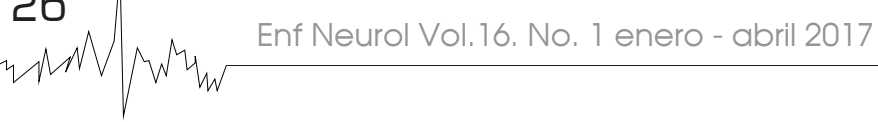




\section{INTRODUCCIÓN}

P or la labor que realiza el personal de enfermería se le puede considerar un grupo vulnerable de sufrir daños a su salud, debido a las situaciones de su actividad profesional generadoras de estrés a las que está expuesto diariamente como lo es la confrontación con la muerte, el sufrimiento de las personas a su cuidado así como las demandas emocionales de los pacientes y familiares entre otros, aunado a ello los posibles conflictos con el personal interdisciplinario como son médicos, supervisores y otras enfermeras.

Las situaciones de estrés, son aquellos eventos a los que estamos expuestos los cuales representan una amenaza o detectamos como peligrosos y no logramos adaptarnos; cuando nuestro organismo no consigue adaptarse, se ve comprometida la salud.

El estrés surge cuando existe una situación que la persona detecta como amenaza hacia su zona de confort lo cual evita que pueda desenvolverse en su vida diaria sin riesgo, las fases de estrés son tres, la primera es la fase de alarma donde el organismo detecta una situación de cambio y pone toda su atención a ella para lograr enfrentarla, si la persona se adapta, el estrés desaparece, la segunda es la fase de resistencia aquí el estresor se mantiene, la persona debe luchar para lograr adaptarse, de lo contrario la resistencia se quiebra ocasionando un desequilibrio en su bienestar físico, mental y social, sí esta fase la supera y consigue la adaptación el estresor desaparece, si no se continua hacia la tercera fase de agotamiento donde el estresor persiste, la energía de la persona se agota y pierde la capacidad para adaptarse, lo que la lleva a un excesivo desgaste tanto psicológico como físico, Barreto menciona que la persona empiezan a tener síntomas físicos y emocionales, como son cefalea, mialgias, insomnio, pesadillas, alteraciones gástricas como gastritis, colitis, ulcera estomacal de origen nervioso, cansancio, fatiga y debilidad, falta de concentración, tristeza excesiva y mal desenvolvimiento de las relaciones interpersonales.'
El Centro para el Control y la Prevención de Enfermedades (CDC) menciona que el estrés laboral aparece cuando las exigencias del trabajo no se igualan con las capacidades del trabajador y esto puede provocar un daño a su salud. ${ }^{2}$ Entendiendo por salud el completo bienestar biológico, psicológico y social y no solo la ausencia de enfermedad, definida por la OMS desde 1948. ${ }^{3}$

En estudios hechos por la Organización Mundial de la salud se reconoce que el 75\% de los trabajadores mexicanos padece de estrés laboral, colocando a México como el principal país con este padecimiento, en segundo lugar a China y en tercer lugar a Estados Unidos. ${ }^{4}$

La Facultad de Medicina de la Universidad Autónoma de México realizó un estudio donde se demuestra que entre el 30\% y 60\% del personal de salud padece de estrés laboral lo cual lo incapacita en la realización adecuada de sus funciones. ${ }^{5}$

Autoridades de enfermería del Hospital Juárez de México, mencionan que "en diferentes estudios realizados en diversas partes del mundo, entre el 80 y el 90 por ciento de las enfermeras se perciben como un personal estresado. Por esta situación, un sector de la población considera que son frías, duras e insensibles en el trato de los enfermos y sus familiares" 6

En el proyecto de investigación "Disminución de los Niveles de Estrés Laboral en Enfermeras que Trabajan en un Hospital Público de Tercer Nivel" realizado por el Hospital Juárez de México en colaboración con el Instituto Politécnico Nacional se afirma que las enfermeras ocupan el sexto lugar de los profesionistas con mayor estrés, lo que causa disminución en su rendimiento y de la calidad de la atención que brindan?

En México el personal de enfermería que actualmente cubre los servicios hospitalarios es insuficiente, por cada 1000 habitantes hay 2.6 enfermeras esto se encuentra por debajo del estándar de la Organización para la Cooperación y el Desarrollo

Enf Neurol Vol. 16. No. 1 enero - abril 2017 
Económicos (OCDE) el cual es 8.88, esto aumenta la carga de trabajo para el profesional de enfermería y no les permite brindar la atención adecuada que requieren los pacientes.

Los accidentes yenfermedades relacionadas al trabajo ponen en riesgo la salud del trabajador pudiendo llegar a ocasionar su muerte. La Organización Internacional del Trabajo (OIT) tiene como objetivo mantener la seguridad y salud de los trabajadores, manteniendo como premisa "trabajo decente es trabajo seguro." "

El doctor Franciso Becerra, subdirector de la Organización Panamericana de Salud (OPS), mencionó durante el seminario celebrado El 28 de abril de 2016 en Washinton D.C. con motivo de la celebración del Día Mundial de la Seguridad y el Trabajo, que "El estrés laboral puede dañar nuestra salud, mermar nuestra productividad y afectar nuestros círculos familiares y sociales."10 Durante la reunión se mencionaron los efectos del estrés laboral sobre la salud como lo son, las cardiopatías, la hipertensión arterial, el evento vascular cerebral, la depresión, trastornos de ansiedad, problemas musculo esqueléticos, así como un aumento en el consumo de alcohol, drogas y tabaco, además de trastornos del sueño y la predisposición a una vida sedentaria. La doctora Julieta Rodríguez, asesora regional en salud de los trabajadores de la OPS/OMS dijo que "estos problemas son capaces de reducir la motivación, el compromiso y el rendimiento laboral." "11 En dicha reunión se recomendó a los países colocar al estrés laboral dentro los padecimientos que pueden afectar al trabajador. ${ }^{12}$

\section{OBJETIVO}

dentificar cuáles son los agentes estresores laborales más comunes a los que se enfrenta el personal de enfermería operativo de las áreas de hospitalización, así como conocer si su nivel de estrés es bajo, moderado o alto.

\section{METODOLOGÍA}

studio de tipo transversal y descriptivo, el
universo lo conformaron 101 enfermeras
del Instituto Nacional de Neurología y
Neurocirugía Manuel Velasco Suárez, el cual
brinda atención de tercer nivel.

Cada enfermero cumplió con los criterios de inclusión los cuales fueron: ser personal de enfermería del turno matutino, vespertino y nocturno, que preste atención directa a los pacientes que se encuentran en los servicio de hospitalización de neurología, neurocirugía, cuarto piso y unidad de psiquiatría.

Los criterios de exclusión incluyeron al personal de enfermería suplente y/o rotatorio, jefes, supervisores y/o coordinadores del servicio, pasantes y estudiantes de enfermería.

Los criterios de inclusión y exclusión fueron escogidos debido a que en las áreas de hospitalización es donde se forma una relación más estrecha enfermera paciente debido a que existe una mayor convivencia y esto se debe a que el paciente la mayoría de las veces aún mantiene una interacción con su entorno y con las personas que lo rodean, además que en esta área es donde por lo general permanece más tiempo hospitalizado hasta su alta médica y retorno a su domicilio, lo cual le permite a la enfermera formar vínculos. Por dicho motivo el estudio solo estuvo dirigido a enfermeras que brindaran atención directa al paciente y que fueran personal adscrito del servicio.

Las variables sociodemográficas que se midieron fueron: edad, sexo, estado civil, escolaridad y presencia de hijos.

Las variables demográficas laborales que se tomaron en cuenta para el estudio fueron: turno en que labora, servicio en el que labora, promedio de número de pacientes que tiene a su cuidado, tiempo laborando en el servicio actual y antigüedad en el Instituto.

\section{8}

MMN Why Enf Neurol Vol. 16. No. 1 enero - abril 2017 
Para el estudio se utilizó un instrumento autoaplicable conformado por 32 reactivos. el cual para su elaboración se tomó en cuenta la versión adaptada al castellano de The Nursing Stress Scale elaborada por Gray y Anderson ${ }^{13}$ y su versión adaptada por Herrera y Cassals $^{14}$ que mide la presencia de estresores laborales en el personal de enfermería, la cual se adecuó e implemento para ser aplicada en un hospital de tercer nivel de atención de la Ciudad de México.

El instrumento está formado por 32 îtems los cuales son contestadas de acuerdo a una escala tipo linkert donde, (0) Nunca, (1) Ocasionalmente, (2) Frecuentemente y (3) Muy frecuentemente, se sumó la puntuación de cada ítem, cuyo rango se encuentra entre 0 y 96, de forma que a mayor puntuación mayor nivel de estrés.

El nivel de estrés se clasifico de la siguiente manera:

- Bajo: $\leq 32$

- Moderado: 33-64

- Alto: $\geq 65$

Los reactivos del instrumento están divididos en ocho factores:

- Factor I. Agonía y muerte

- Factor II. Preparación inadecuada

- Factor III. Carencia de sostén

- Factor IV. Incertidumbre respecto al tratamiento

- Factor V. Relación con los médicos

- Factor VI. Conflicto con otros enfermeros

- Factor VII. Sobrecarga de trabajo

- Factor VIII. Vulnerabilidad

De acuerdo al promedio de las puntuaciones obtenidas de todos los participantes, en cada factor se podrá obtener máximo una media de 3 y mínimo una media de 0 de forma que los reactivos que se acerquen a la media más alta serán los principales factores estresores. De igual forma el promedio obtenido de las puntuaciones de todos los participantes por cada ítem se podrá obtener máximo una media de 3 y mínimo una media de 0, de forma que los reactivos que se acerquen a la media más alta serán los principales estresores laborales.

La aplicación del instrumento se llevó a el 5 de abril de 2016 con previa autorización de cada uno de los jefes de servicio del turno matutino, vespertino y nocturno, de las áreas de hospitalización correspondientes a los servicios de neurocirugía, neurología, cuarto piso y psiquiatría, además se les pidió su apoyo para hacer llegar la invitación al personal de participar informándoles que era de manera voluntaria y anónima, los jefes indicaron el momento más pertinente para llevarlo a cabo y que no afectara la realización de las actividades del personal debido a que el instrumento se contestó en la misma área de trabajo y tomó una duración de 5 a 10 minutos su contestación.

\section{RESULTADOS}

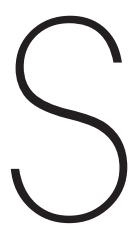

e invitaron a participar a 101 profesionales de enfermería del turno matutino, vespertino y nocturno de las áreas de hospitalización, de los cuales al final sólo 50 contestaron como la abstención del personal de participar en el estudio y la captación de los participantes en un mismo momento pues se encontraban realizando sus funciones lo cual les imposibilitaba contestar la encuesta.

El 58\% de los participantes del estudio corresponde al sexo femenino, la media de edad fue de 41 años, el 48\% está casado el 28\% es soltero y $24 \%$ corresponde a otros estados civiles, del personal encuestado el 68\% refiere la presencia de hijos.

De acuerdo a la escolaridad el 38\% refiere tener nivel de estudios superiores, mientras el 24\% tiene como último nivel de estudios un posgrado, frente al $28 \%$ con una escolaridad a nivel técnico y el 10\% que no contestó. 
48\% del personal tiene menos de 19 años trabajando en el Instituto, frente al 50\% que tiene más de 19 años de antigüedad y el 2\% que no contestó.

El 54\% del personal tiene menos de 6 años laborando en el servicio actual, de 6 a 10 años un $12 \%$, de 11 a 15 años el 16\% y un $18 \%$ corresponde a los que llevan trabajando 16 años o más en el mismo servicio.

El 66\% del personal de enfermería tiene a su cuidado de 4 a 6 pacientes, de 7 a 10 pacientes un 16\%, el 8\% más de 10 pacientes, de 1 a 3 pacientes un 6\% y el $4 \%$ que no contestó

El 52\% de los enfermeros tiene un nivel de estrés moderado siendo la media de la puntuación 36.46, el $46 \%$ tiene un nivel de estrés bajo frente al $2 \%$ que tiene un nivel de estrés alto.

Los cinco factores estresores con medias más altas fueron el factor VIII con media de 1.43, factor I con media de 1.33, factor VII con media de 1.24, factor $\mathrm{V}$ con media de 1.23 y factor II con media de 1.15.

Los tres agentes estresores con medias más altas fueron en primer lugar realizar procedimientos dolorosos para el paciente con una media de 1.88, en segundo lugar mirar sufrir a un paciente y terminar adolorido después de una jornada de trabajo con una media de 1.82, en tercer lugar carencia de personal adecuado para cubrir la unidad con una media de 1.7.

En la prueba de independencia (chi cuadrado) se encontraron diferentes asociaciones entres los agentes estresores, los agentes estresores que están significativamente asociados al aumento de estrés al personal de enfermería fueron los siguientes:

- Conflicto con los médicos y la cantidad de pacientes que el personal de enfermería $(p=0,021)$.

- Exceso de crítica de los supervisoresy la cantidad de pacientes que el personal operativo de enfermería tiene a su cuidado ( $p=0,000)$.
- Indicación médica que parece ser inapropiada para el correcto tratamiento del paciente y el servicio de hospitalización ( $\mathrm{p}=0.034$ ).

- Exceso de crítica por parte de los supervisores y el servicio de hospitalización ( $\mathrm{p}=0,008)$.

- Exceso de crítica por parte de los médicos y el servicio de hospitalización ( $p=0,039)$.

- Carencia del personal adecuado para cubrir la unidad y el servicio de hospitalización ( $\mathrm{p}=0.028$ )

El servicio de neurología en el turno vespertino con una media de 2.67 es el que tiene mayor carencia de personal adecuado, siguiéndole con una media de 2.25 el servicio de neurocirugía en el turno nocturno y el turno matutino del servicio de neurología.

\section{DISCUSIÓN}

L ste estudio demostró que la vulnerabilidad es la principal causa de estrés laboral en este rubro se incluyen repercusiones físicas como terminar adoloridos después de una jornada de trabajo, a diferencia del estudio de Herrera y Cassals $^{15}$ donde la agonía y muerte de las personas es la principal causas de estrés del profesional enfermero, Castillo I, Torres N, Ahumada A, Cárdenas K, Licona $S^{16}$ evidencian que las variables sociodemográficas como la edad, el estado civil y la presencia de hijos influyen al estrés laboral de enfermería, distinto a este estudio donde se observó que las variables que influyen en el estrés laboral son las concernientes al servicio de hospitalización donde se encuentra laborando el personal y el número de pacientes que tienen a su cuidado.

El personal de enfermería tuvo un nivel de estrés moderado con una puntuación promedio de (36.46) similar al estudio de Garza H, Melén dez M, Castañeda H, Aguilera P, Acevedo P, Rangel T con un nivel de estrés laboral de (33.34),17 el nivel de estrés moderado en este estudio se puede atribuir a que al ser realizado en una institución de tercer nivel de atención, el tipo de pacientes al que se le brinda el servicio de salud 
son casos de alta complejidad, el paciente demanda más cuidado y atención, además que requiere de cuidados específicos como especializados.

Aunque los resultados que se obtuvieron en esta investigación dan un conocimiento más amplio sobre las situaciones que causan estrés laboral al personal de enfermería y en específicamente del Instituto Nacional de Neurología y Neurocirugía, no se puede generalizar a toda la población del hospital debido a que la muestra finita que se necesitaba para conocer el nivel de estrés y los agentes estresores de mayor prevalencia era de $n=81$ enfermeros la cual para calcularla se utilizó ( $\left.z_{a}=1.96\right)$ para un nivel de confianza del 95\%. Además que se presentaron ciertas limitaciones en la realización de esta investigación las cuales son concernientes a la aplicación del instrumento, hubo complicaciones para que todos los enfermeros pudieran contestarlo debido a que se realizó en un solo momento y en su lugar de trabajo lo cual interfería en sus labores, además que algunos participantes refirieron que el instrumento era muy extenso y las preguntas complejas, lo cual no garantiza que se haya contestado correctamente.

\section{CONCLUSIONES}

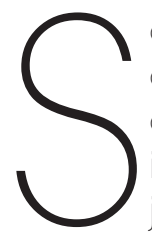
on diferentes estresores laborales a los que el personal de enfermería se enfrenta cada día, consecuencia de la actividad e intervenciones que desempeña durante su jornada de trabajo; para lograr alcanzar el alta del paciente para que esté pueda iniciar su rehabilitación y tener mejor calidad de vida con los capacidades con que cuente el enfermo y los recursos de la familia, así como prevenir la aparición de complicaciones que puedan causarle riesgos o dejarle un daño permanente e incluso la muerte.
En este estudio se logró identificar los agentes estresores laborales más comunes así como el nivel de estrés al que está expuesto el personal de enfermería de las áreas de hospitalización, el cual es producto de las situaciones que vive a diario el profesional de enfermería para realizar sus tareas a favor del paciente esto lo expone a tener un nivel de estrés moderado, lo cual podría perjudicar su salud, pudiendo provocar una disminución en el desempeño sobre la calidad de las tareas que se realizan.

Cumpliendo la hipótesis de que existe una fuerte relación entre el hacer profesional de enfermería y el estrés laboral, se propone utilizar el modelo de investigación del Hospital Juárez de México en donde se realizó un estudio específico con el objetivo de disminuir el nivel de estrés en el personal de enfermería en un hospital de tercer nivel de atención, previamente la exposición al estrés se detectó a través de la aplicación de cuestionarios y la medición del cortisol hormona que se libera ante situaciones de estrés, las intervenciones que propusieron para alcanzar el objetivo son a través de diferentes terapias como la acupuntura y sus variantes, la farmacología y la orientación psicológica. ${ }^{18}$

Se pretende que este trabajo sirva como fuente de consulta para motivar la investigación en salud ocupacional en enfermería y que sirva de línea de investigación para el estudio de las diferentes asociaciones con el estrés como puede ser medir el desempeño laboral, capacidades de adaptación y percepción de calidad de vida, esto con el fin de detectar los principales problemas que existen, sus causas y consecuencias, y de esta manera elaborar estrategias pertinentes que protejan la salud del profesional de enfermería y les permita alcanzar niveles altos de productividad. 
Tabla 1. Lugar que ocupan los estresores laborales más comunes del personal de enfermería

\begin{tabular}{|c|c|c|c|}
\hline & Estresores laborales & $\bar{x}$ & $\begin{array}{l}\text { Desviación } \\
\text { estándar }\end{array}$ \\
\hline 1 & Realizar procedimientos dolorosos para el paciente & 1.88 & 0.75 \\
\hline 2 & $\begin{array}{l}\text { Mirar sufrir a un paciente y terminar adolorido después de } \\
\text { una jornada de trabajo }\end{array}$ & 1.82 & 1.04 \\
\hline 3 & Carencia de personal adecuado para cubrir la unidad & 1.72 & 1.11 \\
\hline 4 & inadecuada información del médico sobre el paciente & 1.56 & 0.93 \\
\hline 5 & Dormir menos de 6 horas cuatro noches a la semana & 1.5 & 1.09 \\
\hline 6 & $\begin{array}{l}\text { Ser interrogado por el paciente sobre cuestiones que no } \\
\text { poseo una respuesta satisfactoria }\end{array}$ & 1.4 & 0.90 \\
\hline 7 & $\begin{array}{l}\text { Indicación médica que parece ser inapropiada para el } \\
\text { correcto tratamiento del paciente }\end{array}$ & 1.32 & 0.89 \\
\hline 8 & Dormir y no descansar & 1.3 & 0.91 \\
\hline 9 & $\begin{array}{l}\text { Carencia de tiempo para ofrecer soporte emocional al } \\
\text { paciente }\end{array}$ & 1.28 & 0.95 \\
\hline 10 & $\begin{array}{l}\text { Tomar una decisión concerniente al paciente cuando el } \\
\text { médico no se encuentra de imediato disponible }\end{array}$ & 1.24 & 0.56 \\
\hline
\end{tabular}

Tabla 2. Factores estresores presentados por orden de importancia

\begin{tabular}{|c|c|c|}
\hline Factores estresores & $\bar{x}$ & $\begin{array}{l}\text { Desviación } \\
\text { estándar }\end{array}$ \\
\hline Factor VIII Vulnerabilidad & 1.43 & 0.97 \\
\hline Factor I, Agonía y muerte & 1.33 & 0.88 \\
\hline Factor VII. Sobrecarga de trabajo & 1.24 & 1.03 \\
\hline Factor IV. Incertidumbre respecto al tratamiento & 1.23 & 0.93 \\
\hline Factor II. Preparación inadecuada & 1.15 & 0.87 \\
\hline Factor III. Carencia de sosten & 0.99 & 0.87 \\
\hline Factor V. Relación con los médicos & 0.92 & 0.81 \\
\hline Factor VI. Conflicto con otros enfermeros & 0.76 & 0.86 \\
\hline
\end{tabular}


Tabla 3. Factores por orden de importancia y el agente estresor que más influye en ese factor

Principales factores y agentes estresores que influyen en el estrés laboral de enfermería

\begin{tabular}{ll|l} 
Factores estresores & $\bar{X}$ & Agente estresor \\
\hline
\end{tabular}

Tabla 4. Carencia de personal adecuado para cubrir la unidad

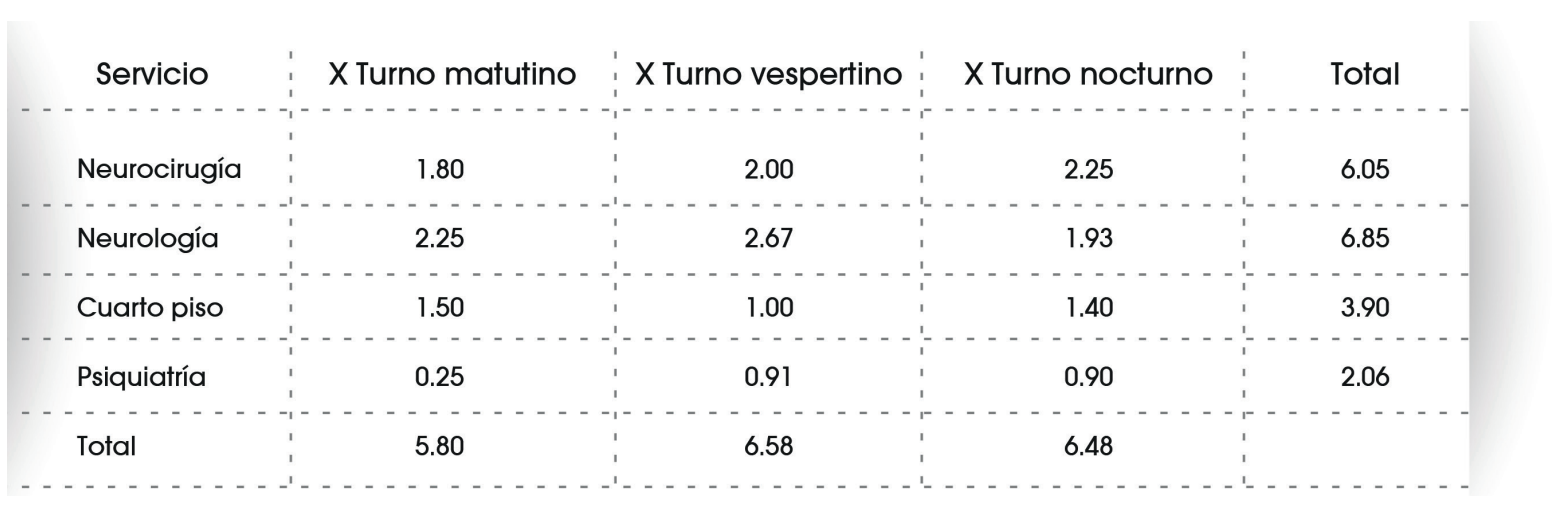


Figura 1. Relación entre diferentes agentes estresores y el servicio de hospitalización Indicación médica que parece ser inapropiada para el correcto tratamiento del paciente

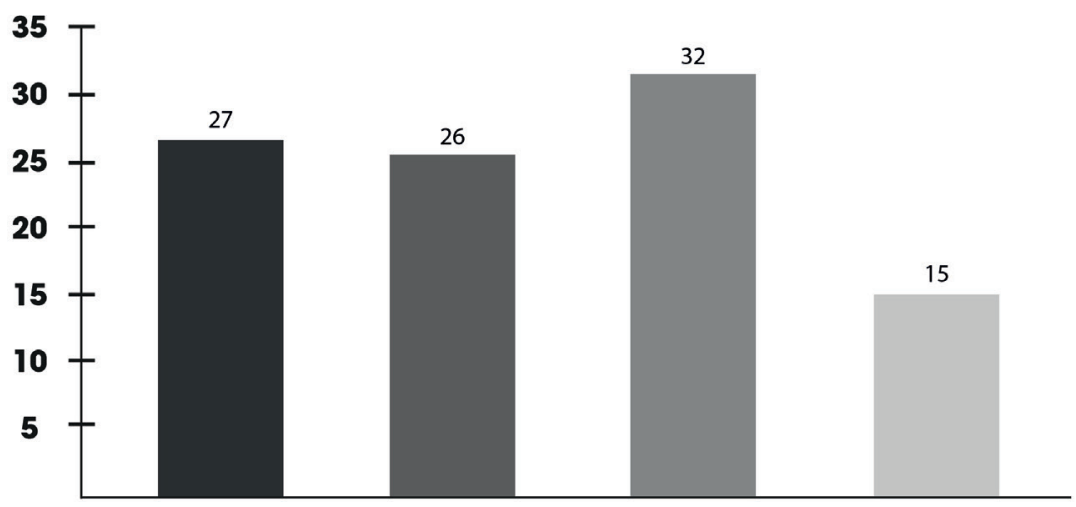

Neurocirugía

Neurología

Cuarto piso

Psiquiatría

Exceso de crítica por parte de los supervisores

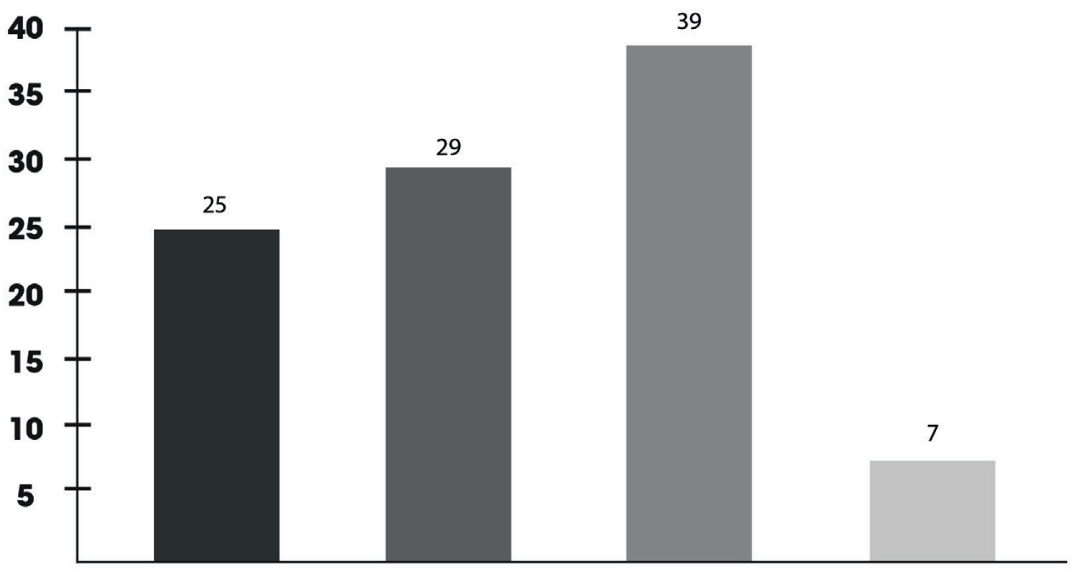

Neurocirugía

Neurología

Cuarto piso

Psiquiatría

Exceso de crítica por parte de los médicos

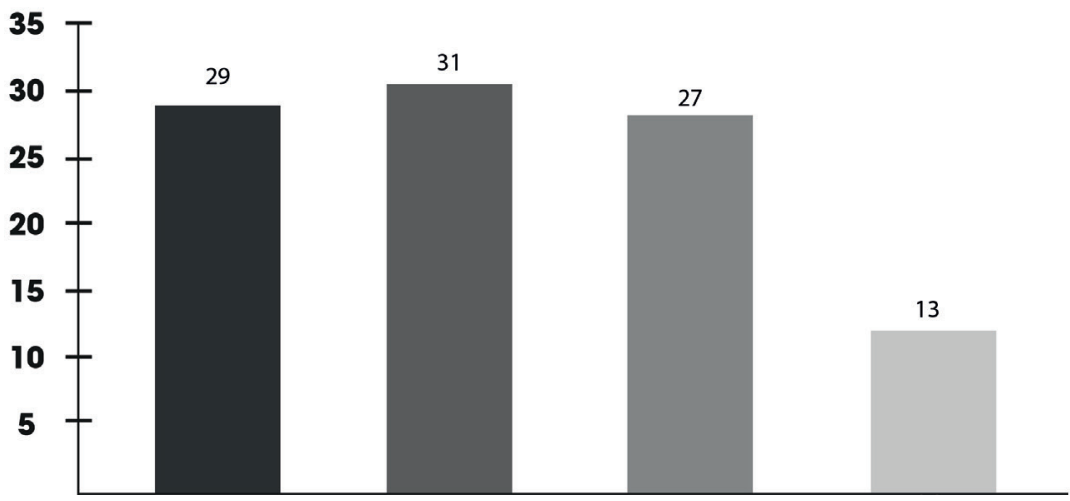




\section{REFERENCIAS BIBLIOGRÁFICAS}

1. Herrera R, Cassals M. Algunos factores influyentes en la calidad de vida laboral de enfermería. Rev Cubana Enfermer (serie en Internet) 2005 (consultado el 11 de agosto de 2015); 21(1): 1-1. Disponible en: http://bit. Iy/1VSUG08

2. Centro para el control y la prevención de enfermedades (sitio de internet). colc.gov intOnline Resources, Inc.; 2015(actualizado el 21 de enero de 2015; (consultado 9 de septiembrede 2015). Disponible en: http://1.usa. gov/1 NcH740

3. Organización Mundial de la Salud (sitio de internet). who.int (consultado 13 de septiembre de 2015). Disponible en: http://bit.ly/1VRUSsqA

4. El Universal (sitio de internet) eluniversal.com.mx (consultado 22 de agosto de 2016). Disponible en: http://e/unimx/2buOnRu

5. Fundación UNAM (sitio de internet) fundacionunam.org.mx (consultado 23 de agosto de 2016). Disponible en: http://bit.ly/2bDHJEG

6. Hospital Juárez de México Blog Oficial (sitio de internet) hospitaliuarezdemexico.blogspot.mx (consultado 23 de agosto de 2016). Disponible en: http://bit.ly/2bR6FJB

7. Hospital Juárez de México Blog Oficial. Op. Cit

8. Organisation for EconomicCo-operation and Development (sitio de internet) oecd.org (consultado 24 de agosto de 2016). Disponible en: http:// bit.ly/1PrtDVb

9. Organización Internacional del Trabajo (sitio de internet) ilo.org Recursos en línea, 2016 (consultado 24 de agosto de 2016). Disponible en: http://bitt. Iy/1bzuqTD

10. Organización Panamericana de la salud (sitio de internet) paho.org (actualizado el 4 de mayo de 2016; consultado 22 de septiembre de 2016). Disponible en: http://bit.ly/2bR9omb

11. Organización Panamericana de la salud. Op. Cit

12. Organización Panamericana de la salud. Op. Cit.

13. Más P, Escribá A. La versión castellana de la escala, The Nursing Stress Scale, proceso de adaptación transcultural. Rev Esp Salud Pública (serie en Internet) 1998 (consultado el 11 de febrero de 2016); 72:529-538. Disponible en: http://bit.IY/1Q9TGNO
14. Herrera R, Cassals M. Op. Cit.

15. Herrera R, Cassals M. Op. Cit.

16. Castillo I, Torres N, Ahumada A, Cárdenas K, Licona S. Estrés laboral en enfermería y factores asociados. Cartagena (Colombia). Salud Uninorte. Barranquilla (Col.) (serie en Internet) 2014 (consultado el 8 de septiembre de 2015): 30(1):34-43. Disponible en: http://bit.Iy/1RaVqHU

17. Garza H, Meléndez M, Castañeda H, Aguilera P, Acevedo P, Rangel T. Nivel de Estrés en Enfermeras que Laboran en Áreas de Hospitalización Desarrollo Cientif Enferm (serie en internet) 2011 (consultado el 8 de Julio de 2016); 19(1):15-19. Disponible en: http://bittly/2aMXUNj

18. Hospital Juárez de México Blog Oficial. Op. Cit

19. Martínez M, Alba L, Sanabria G. Interrelación entre calidad de vida y motivaciones relativas a la salud. Rev Cubana Med Gen Integr (serie en internet) 2010 (consultado el 14 de octubre de 2015); 26(1):147-159. Disponible en: http://bit.Iy/1VSsBBu

20. Zabalegui A. El rol del profesional de enfermería. AQUICHAN (serie en internet) 2003 (consultado el 30 de agosto de 2015);3(3):16-20. Disponible en hitp://bit.IY/1NTGjTT

21. Gómez O, Sema S, Becerril V. Knaul F, Arreola H, Frenk J. Sistema de salud de México.Salud Publica Mex (serie en internet) 2011 (consultado el 30 de agosto de 2015); 53(2):220-232. Disponible en: http://bit.ly/10w4d9T

22. Garza H, Meléndez M, Castañeda $H$, Aguilera P, Acevedo P, Rangel T, Nivel de Estrés en Enfermeras que Laboran en Áreas de Hospitalización. Desarrollo Cientif Enferm (serie en internet) 2011 (consultado el 8 de Julio de 2016): 19(7):15-19. Disponible en: http://bit.ly/2aMXUNj

23. Senado J. Los factores de riesgo en el proceso salud enfermedad Rev Cubana Med Gen Integr (serie en internet) 1999 (consultado el 24 de agosto de 2015); 15(4):453-60. Disponible en: http://bit.ly/1k4J7m5

24. Barreto J, Augusta M, Das Neves M, Pagliarini M, Silva S. Estrés y recursos de enfrentamiento del cuidador en el contexto hospitalario. Salud Mento (serie en Internet) 2017 (consultado el 13 de septiembre de 2015); 34(2):129 138. Disponible en: http://bit.ly/1GH93sP 